\title{
Polyinosinic:polycytidylic acid aggravates calcipotriol-induced atopic dermatitis-like skin lesions in mice by increasing the expression of thymic stromal lymphopoietin
}

\author{
Haoyue Wan ${ }^{\wedge}$, Huixue Yang^^, Mingjing Wei^, Wenqi Chen ${ }^{\wedge}$ \\ Department of Dermatology, Nanjing First Hospital, Nanjing Medical University, Nanjing, China \\ Contributions: (I) Conception and design: W Chen, H Wan; (II) Administrative support: W Chen; (III) Provision of study materials or patients: H \\ Wan, H Yang, M Wei; (IV) Collection and assembly of data: H Wan, H Yang; (V) Data analysis and interpretation: Ha Wan, H Yang, M Wei; (VI) \\ Manuscript writing: All authors; (VII) Final approval of manuscript: All authors. \\ Correspondence to: Wenqi Chen. Department of Dermatology, Nanjing First Hospital, Nanjing Medical University, Changle Road, Nanjing 210006, \\ China. Email: 1009434376@qq.com.
}

Background: Polyinosinic:polycytidylic acid [poly (I:C)] is a synthetic viral double-stranded RNA analog
that can activate Toll-like receptor 3 (TLR3) and induce the release of thymic stromal lymphopoietin (TSLP).
TSLP has been shown to contribute to atopic dermatitis (AD). This study explored the effects of poly (I:C)
in a calcipotriol-induced model of murine AD.

Methods: Calcipotriol (MC903) was used to establish AD-like mice model. Mice in the MC903 + poly (I:C) group were then treated with poly (I:C) in a concentration of $5 \mu \mathrm{g} / \mathrm{g}$ bodyweight. The impact of poly (I:C) treatment on these animals was assessed based upon changes in lesions, bodyweight, ear thickness, and histopathological findings. In addition, serum interleukin 4 (IL-4), interferon- $\gamma$ (IFN- $\gamma$ ), immunoglobulin E (IgE), IL-13, and TSLP levels were measured using enzyme-linked immunosorbent assay (ELISA), while tissue IL-13 and TSLP levels were assessed using ELISA, Western blotting, and immunohistochemical staining, and mast cell infiltration was assessed through toluidine blue (TBO) staining.

Results: Relative to vehicle control treatment, poly (I:C) administration was associated with a significant exacerbation of calcipotriol-induced AD-like murine skin lesions. In animals treated with poly (I:C), the levels of serum IL-4, IL-13 and TSLP increased significantly, while the level of IFN- $\gamma$ did not change. It also increased IL-13 and TSLP levels in skin lesions relative to the control-group mice and increased dermal mast cell infiltration and $\operatorname{IgE}$ production.

Conclusions: These data indicate that poly (I:C) treatment and exogenous activation of TLR3 exacerbate murine calcipotriol-induced AD-like skin lesions in part by increasing the production of TSLP and other T-helper 2 (Th2)-related cytokines.

Keywords: Atopic dermatitis (AD); polyinosinic:polycytidylic acid [poly (I:C)]; thymic stromal lymphopoietin (TSLP); Toll-like receptor 3 (TLR3)

Submitted Dec 22, 2021. Accepted for publication Feb 18, 2022.

doi: $10.21037 /$ atm-22-282

View this article at: https://dx.doi.org/10.21037/atm-22-282

\footnotetext{
^ ORCID: Haoyue Wan, 0000-0001-7494-7924; Huixue Yang, 0000-0003-4259-8832; Mingjing Wei, 0000-0003-0866-6889; Wenqi Chen, 0000-0003-4552-541X.
} 


\section{Introduction}

Atopic dermatitis (AD) is a common chronic inflammatory skin disease that results in intense itching, dry skin, and recurrent eczema (1). $\mathrm{AD}$ is generally associated with other immunoglobulin E (IgE)-related disorders, including food allergies, asthma, and allergic rhinitis (2). The etiological basis for $\mathrm{AD}$ is complex and includes genetic factors, abnormalities within the microbiome, neuroimmune interactions, impaired epidermal barrier functionality, and cutaneous inflammatory responses, all of which can result in epidermal and immunological abnormalities (3). The T-helper 2 (Th2) pathway arm of the adaptive immune response is currently thought to be the primary driver of AD-related pathology, with substantial contributions from the Th1, Th17, and Th22 axes and from the JAK/ STAT signaling pathways (4-7). Interleukin (IL)-4 and IL13 are key Th2 cytokines thought to be integral to AD development and progression $(8,9)$, with recent evidence suggesting IL-13 is the primary Th2 cytokine responsible for driving peripheral inflammation $(10,11)$. Consistently, biologic drugs that target IL-13, including the anti-IL$4 \mathrm{R} \alpha$ antibody dupilumab and the anti-IL-13 antibody tralokinumab, play key roles in $\mathrm{AD}$ lesion resolution (12-14). Prior work has shown that the vitamin $\mathrm{D}$ receptor ligand calcitriol $\left[1 \alpha, 25(\mathrm{OH})_{2} \mathrm{D}_{3}\right]$ or its low-calcemic analog calcipotriol (MC903) can promote the expression of thymic stromal lymphopoietin (TSLP) by epidermal keratinocytes, resulting in a syndrome similar to $\mathrm{AD}(15,16)$. Topical MC903 application can also alter skin morphology, inducing inflammation and elevations in levels of serum IgE similar to those observed in patients with acute extrinsic $\mathrm{AD}$ (17). To further explore this pathological context, we developed calcipotriol-induced AD-like skin lesion model mice.

TSLP is a pleiotropic cytokine expressed primarily by fibroblasts, keratinocytes, intestinal epithelial cells, and lung epithelial cells following activation, although it can also be produced by certain immune cell types such as mast cells and dendritic cells (DCs) (18). TSLP is often regarded as a so-called "alarmin", as it can be released from epithelial barriers in response to external stressors and has been shown to contribute to allergy-related inflammatory responses (19). TSLP can condition DCs to upregulate OX40 ligand (OX40L), thereby promoting the OX40L signaling-mediated differentiation of inflammatory Th2 cells that secrete cytokines, including IL-4, IL-5, IL-13, and tumor necrosis factor- $\alpha$ (TNF- $\alpha)(20,21)$. Keratinocytederived TSLP can activate the TSLP receptor complex in afferent sensory neurons to induce AD-related pruritus (22). Inhibiting the production of TSLP can markedly alleviate such pruritus and improve the quality of life in patients with AD (23). Some reports suggest that TSLP can promote skin inflammation by activating basophils, although further research will be needed to fully clarify the roles of basophils and TSLP in this pathological context (24). Tezepelumab is a first-in-class human monoclonal antibody specific to TSLP that is currently being assessed as a potential treatment for $\mathrm{AD}$ (25). Toll-like receptor 3 (TLR3) is an innate immune pattern recognition receptor (PRR) that primarily recognizes double-stranded RNA (dsRNA) derived from bacteria, viruses, or damaged cells. Subsequently, it can activate a range of downstream signaling pathways that are important in infectious and oncological contexts (26). However, the role of TLR3 in the occurrence and development of $\mathrm{AD}$ still remains unclear. Polyinosinic:polycytidylic acid [poly (I:C)] is a synthetic viral dsRNA analog that can activate TLR3. When poly (I:C) is used to treat keratinocytes, chemokines and cytokines are released, including TSLP, IL- 6 , TNF- $\alpha$, interferon- $\beta$ (IFN- $\beta$ ), and granulocyte-macrophage colony-stimulating factor (GM-CSF) $(27,28)$. Extracellular dsRNA can also induce the release of TSLP through NF- $\mathrm{NB}$-dependent mechanisms $(29,30)$.

Given the above evidence, it is likely that TLR3 activation and increased TSLP expression may result in adverse immunopathological outcomes in murine $\mathrm{AD}$ models. To test this possibility, we analyzed $\mathrm{AD}$ model mice and found that poly (I:C) could promote the expression of TSLP, IL-13, and IL-4 together with concomitant increases in $\mathrm{IgE}$ production and mast cell dermal infiltration, resulting in the aggravation of skin lesions. Together, these data offer new insights into the potential mechanisms whereby TLR3 can influence $\mathrm{AD}$ pathogenesis, highlighting new avenues for future clinical diagnostic and treatment strategies. We present the following article in accordance with the ARRIVE reporting checklist (available at https:// atm.amegroups.com/article/view/10.21037/atm-22-282/rc).

\section{Methods}

\section{Animals}

Male BALB/c mice (6-week-old, $24 \mathrm{~g}$ ) were obtained from Vital River, Inc. (Beijing, China) and housed under specific pathogen-free conditions. Five mice were housed in each cage in a humidity and temperature-controlled facility with 
A



B

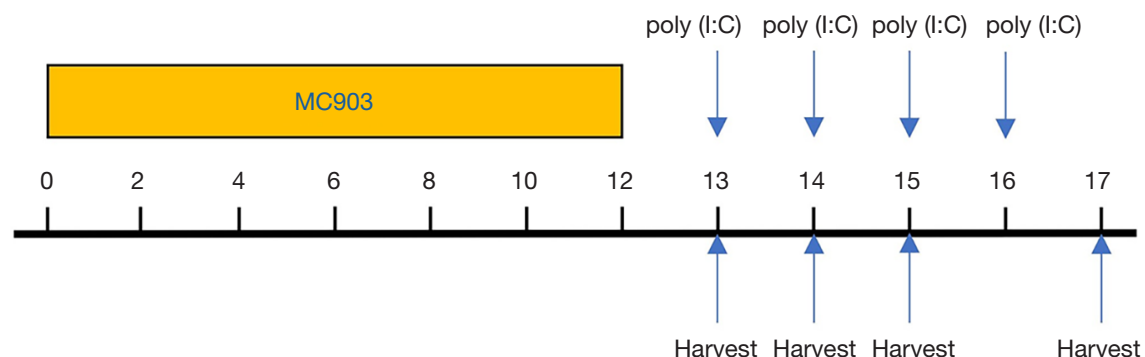

Figure 1 Overview of the treatment schedules for the calcipotriol (MC903)-induced atopic dermatitis (AD)-like skin lesions model in experimental mice. Topical MC903 application was performed from days 0 to 12, with polyinosinic:polycytidylic acid [poly (I:C)] applied on days 13 and 14. On day 15, the experiment was terminated (A). For the other experiments, an AD mouse model was established as above. Mice were separated into 4 groups and injected daily with poly (I:C) for 0 to 4 days (B). Poly (I:C) injection and sample collection time points are denoted with arrows.

a 12-hour light/dark cycle. All animal studies were approved by the Institutional Ethics Committee of Nanjing First Hospital, Nanjing Medical University (Project License DWSY-2001134), and all experimentally related processes and animal care followed the institutional ethics directions for animal-related experimental processes. Animals were randomly separated into naïve control (NC), MC903, MC903 + vehicle, and MC903 + poly (I:C) treatment groups with 8-10 mice from multiple litters in each group. Though 60 mice were prepared for this study, only 57 were included. Three mice were excluded because of instrumentation or technical failure during preparation.

\section{AD animal model establishment}

A murine model of AD-like disease was established as in a prior study. ${ }^{17}$ Briefly, mice were administered a daily topical dose of a $15 \mu \mathrm{L} 0.005 \%$ calcipotriol scalp solution (MC903) (170281, LEO Pharma A/S, Ballerup, Denmark), which was applied to the dorsal and ventral sides of each ear for 12 consecutive days. Control animals received $15 \mu \mathrm{L}$ of ethanol instead (31). Mice in the MC903 + poly
(I:C) group were then intraperitoneally (i.p.) treated with poly (I:C) (tlrl-pic-5, Invivogen, CA, USA) in a concentration of $5 \mu \mathrm{g} / \mathrm{g}$ bodyweight and assessed 2 days after continuous injection. All operations were conducted following the manufacturer's instructions. Animals in the MC903 + vehicle group were administered an equivalent vehicle dose. Murine bodyweight and ear thickness were measured on days $0,1,3,7,9,10,12,13,14$, and 15. Ear thickness was measured using electronic calipers to gauge the degree of edema, with ear and serum samples being collected at designated time points (Figure 1A). To further assess the impact of poly (I:C) on the expression of TSLP and IL-13 and to better clarify the underlying mechanisms, we conducted Western blotting analyses and ELISAs for samples from our MC903-induced AD-like mice after continuous treatment with poly (I:C) for 0-4 days (Figure 1B). After collection, serum was centrifuged and stored at $-80^{\circ} \mathrm{C}$. Ear samples were separated into 2 parts, 1 of which was stored at $-80{ }^{\circ} \mathrm{C}$ for protein extraction and the other of which was fixed using $4 \%$ paraformaldehyde for subsequent immunohistochemical and histopathological analyses. 


\section{Serum cytokine measurements}

Cytokine levels were measured in murine serum samples using enzyme-linked immunosorbent assay (ELISA) kits specific to murine IL-4 (PI613, Beyotime Inc. Shanghai, China) and IFN- $\gamma$ (PI507, Beyotime Inc. Shanghai, China), ELISA kits specific to murine IgE from CUSABIO, Inc. (CSB-E07983m, Wuhan, China), IL-13 (ab219634, Abcam, Cambridge, MA, USA) and TSLP ELISA kits (ab155461, Abcam, Cambridge, MA, USA). All assays were conducted following the manufacturer's instructions, with samples analyzed in triplicate.

\section{Tissue TSLP and IL-13 level analyses}

Murine ear tissue samples were thawed, minced in a $15 \mathrm{~mL}$ tube, and homogenized in $5 \mathrm{~mL}$ of radioimmunoprecipitation assay (RIPA) buffer supplemented with $10 \mu \mathrm{L}$ protease inhibitors, $10 \mu \mathrm{L}$ phosphatase inhibitors, and $5 \mu \mathrm{L}$ phenylmethylsulfonyl fluoride (PMSF) (Beyotime Inc., Shanghai, China) for 5 minutes at $4^{\circ} \mathrm{C}$. Samples were then centrifuged $\left(12,000 \mathrm{rpm}, 5\right.$ minutes, $\left.4{ }^{\circ} \mathrm{C}\right)$, and supernatants were collected to measure IL-13 and TSLP levels using the ELISA kits described above. Total protein levels were measured with a bicinchoninic acid (BCA) kit (23227, Thermo Fisher Scientific, MA, USA) following the manufacturer's instructions.

\section{Histopathological and immunobistochemical staining}

Murine ear tissue samples were fixed using $4 \%$ formaldehyde, dehydrated with an ethanol gradient, and paraffin-embedded. Hematoxylin and eosin (H\&E), and toluidine blue (TBO) staining of these samples was then conducted to gauge mast cell infiltration, with sections subsequently being visualized via microscopy. In addition, TSLP and IL-13 receptor $\alpha 1$ (IL-13R $\alpha 1$ ) levels in murine ears were assessed via immunohistochemical staining with antibodies specific to TSLP (ab115700, Abcam, Cambridge, MA, USA) and IL-13R $\alpha 1$ (ab79277, Abcam, Cambridge, MA, USA). Samples were then probed at room temperature with a secondary HRP-conjugated goat anti-rabbit IgG antibody (ab205718, Abcam, Cambridge, MA, USA) rinsed with PBS, stained with DAB substrate, and hematoxylin counterstained.

\section{Western blotting}

RIPA buffer was used to lyse mouse ear tissue samples as above, after which protein levels therein were measured via BCA assay. Equal protein amounts were then separated via $6-15 \%$ sodium dodecyl sulfate-polyacrylamide gel electrophoresis (SDS-PAGE) and transferred to polyvinylidene fluoride (PVDF) membranes (Thermo Fisher Scientific, MA, USA). These blots were then blocked for 2 hours using $5 \%$ non-fat milk (Beyotime, Shanghai, China) in $0.1 \%$ Tween 20 in tris-buffered saline (TBS-T) at $26^{\circ} \mathrm{C}$. They were then incubated overnight at $4{ }^{\circ} \mathrm{C}$ while being constantly shaken with primary antibodies specific to $\beta$-actin (HRP-60008, Proteintech, IL, USA), TLR3 (ab62566, Abcam, Cambridge, MA, USA), IL-13Ra1 (ab79277, Abcam, Cambridge, MA, USA), or TSLP (ab115700, Abcam, Cambridge, MA, USA). Blots were then washed repeatedly, probed for 2 hours with secondary horseradish peroxidase (HRP)-conjugated goat anti-rabbit IgG antibody (ab205718, Abcam, Cambridge, MA, USA), with $\beta$-actin being assessed as a loading control. Protein bands were then detected using enhanced chemiluminescence (ECL) detection reagents (Thermo Fisher Scientific, MA, USA) with a Tanon 5200 automatic chemiluminescence image analysis system (Tanon, Shanghai, China).

\section{Statistical analysis}

GraphPad Prism 8.0.2 software (GraphPad Software, CA, USA) was used for data analysis, with a $\mathrm{P}$ value $<0.05$ as the significance threshold. All analyses were conducted with a minimum of 3 replicate samples per group. Data are expressed as means \pm standard deviations (SD). Comparison of continuous information was based on individual $t$-tests between the two groups.

\section{Results}

\section{Evaluation of the impact of poly (I:C) on calcipotriol-induced} AD-like skin lesions

We began by assessing the effects of poly (I:C) on skin lesions in calcipotriol-induced AD-like model mice. The application of calcipotriol (MC903) in isolation resulted in the drying and desquamation of ear tissue with pronounced telangiectasia in treated mice (Figure $2 A$ ). H\&E staining further revealed evidence of increasing epidermal thickness and inflammatory cell infiltration into the dermis in these model mice (Figure 2B). Administration of poly (I:C) was associated with increased immune cell infiltration and epidermal thickness (Figure 2B). Similarly, ear skin inflammation was more exacerbated in mice in the MC903 
A

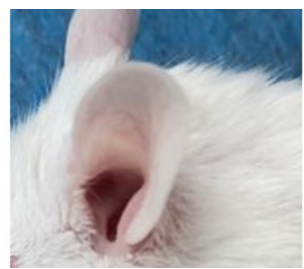

B



NC


MC903

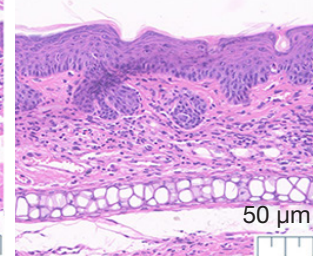

MC903 + vehicle

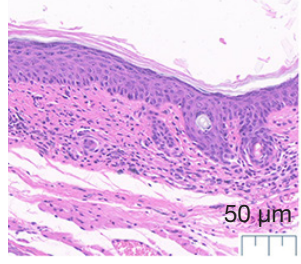

MC903 + poly $(\mathrm{I}: \mathrm{C})$

C
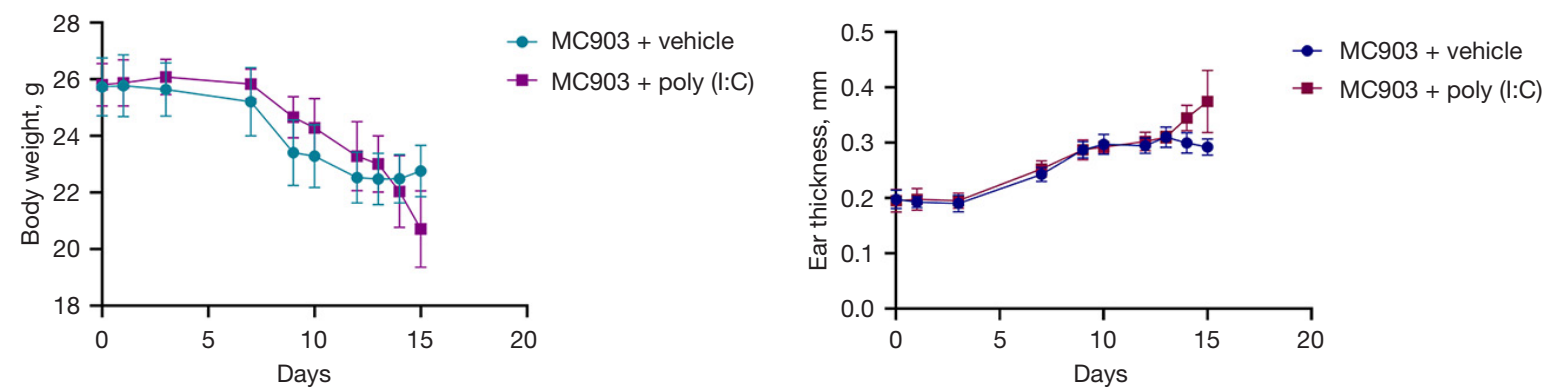

Figure 2 Representative images of the ears of mice in the different treatment groups demonstrating atopic dermatitis (AD)-like cutaneous lesions (A). Representative hematoxylin and eosin (H\&E)-stained epidermal tissue sections from ears of mice in the indicated treatment groups demonstrating epidermal thickness and the infiltration of inflammatory cells into the dermis. Scale bar $=50 \mu \mathrm{m}(\mathrm{B})$. Bodyweight and ear thickness were quantified on days $0,1,3,7,9,10,12,13,14$ and 15 (C,D). $P<0.05$ was the significance threshold, measured by $t$-test.

+ poly (I:C) treatment group, than those in the MC903 + vehicle treatment group, as evidenced by weight loss, itching behavior, and a significant increase in ear thickness (Figure 2C,2D). Together, these data were consistent with the claim that poly (I:C) treatment can exacerbate cutaneous inflammation in mice.

\section{Poly (I:C) exacerbates cutaneous inflammation in AD model mice}

Th2 cytokines have been closely linked to the pathogenesis of AD (32). TSLP, which can be produced in response to the detection of extracellular dsRNA, can stimulate the secretion of IL-4 and IL-13. Therefore, we next explored the effects of poly (I:C) on the secretion of these cytokines in our MC903-induced murine model of AD-like disease.
Serum TSLP, IL-4, and IL-13 levels in NC mice were relatively low but were increased in the serum of mice in the MC903 treatment group. Repeated poly (I:C) exposure was associated with marked increases in the levels of all three of these cytokines, whereas serum IFN- $\gamma$ levels were unchanged (Figure 3A-3D). We additionally assessed levels of total protein, TSLP, and IL-13 in skin lesion tissue samples, revealing that poly (I:C) significantly increased TSLP and IL-13 levels in skin lesions in MC903 + poly (I:C) treatment group compared to levels in the MC903+ vehicle treatment group (Figure $3 E, 3 F$ ). Western blotting similarly confirmed the significant upregulation of TLR3, TSLP, and IL-13R 1 in MC903 + poly (I:C) treatment group (Figure 3G-37), and the frequency of TSLP $^{+}$and IL$13 \mathrm{R} \alpha 1^{+}$cells in cutaneous lesions rose following poly (I:C) treatment (Figure 3K). 
A

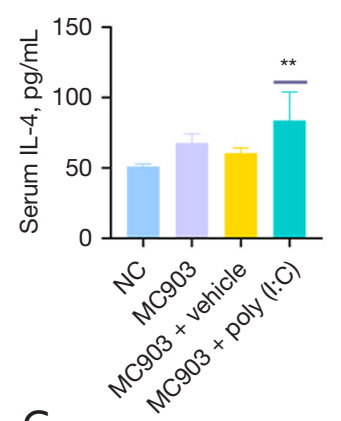

C

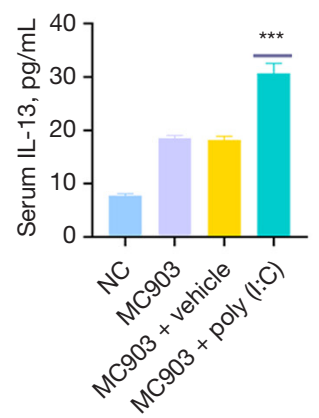

E

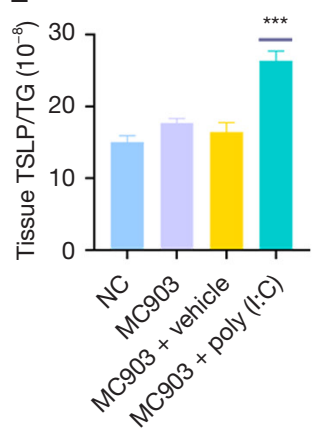

B

D
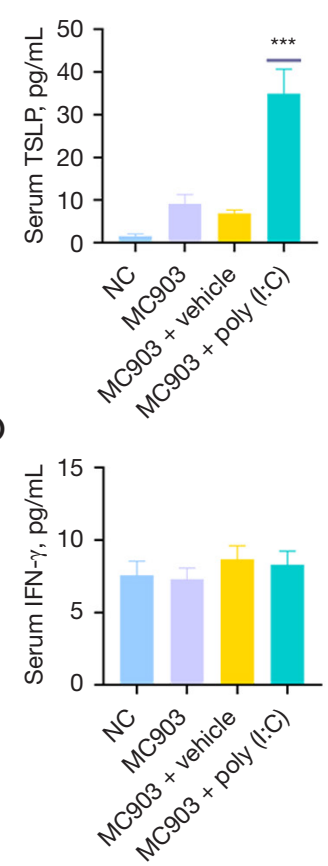

F

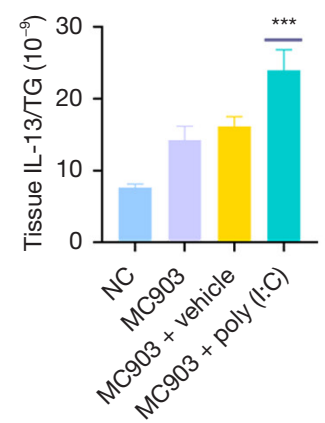

G



I

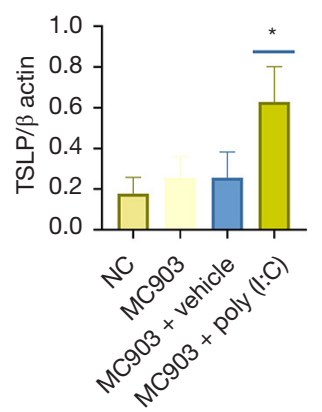

K

TSLP
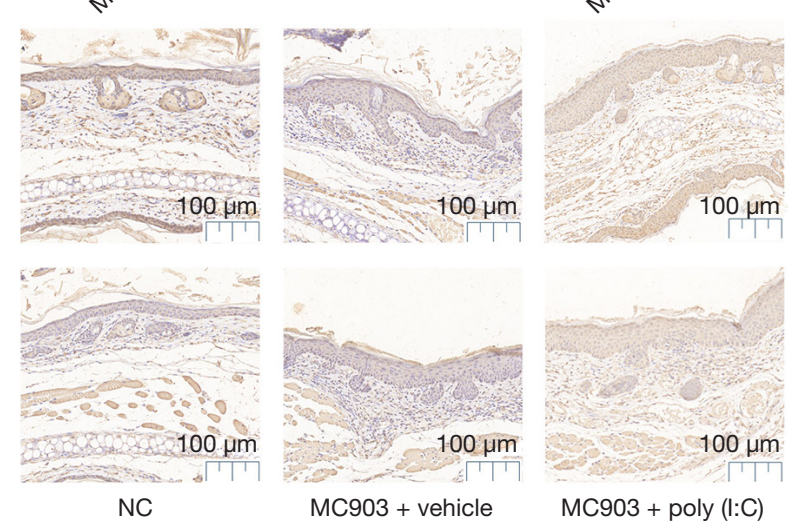

Figure 3 The impact of polyinosinic:polycytidylic acid [poly (I:C)] treatment on serum IL-4, TSLP, IL-13, and IFN- $\gamma$ was assessed in a murine model of MC903-induced atopic dermatitis (AD)-like disease (A-D). Tissue TSLP and IL-13 levels were additionally measured using enzyme-linked immunosorbent assay (ELISA) kits with normalization to total protein concentrations as quantified by bicinchoninic acid (BCA) assay (E,F). Western blotting analyses of TLR3, TSLP, and IL-13R $\alpha 1$ levels (G-J). Densitometric analyses are representative of 3 independent experiments shown in Figure S1. Immunohistochemical analyses of $\mathrm{TSLP}^{+}$and IL-13R $\alpha 1^{+}$cells in tissue sections. Scale bar $=100 \mu \mathrm{m}(\mathrm{K})$. Data are expressed as means $\pm \mathrm{SD}$, in (A-F), $\mathrm{n}=10$ /group, in (G-J), $\mathrm{n}=3 /$ group. ${ }^{*}, \mathrm{P}<0.05,{ }^{* *}, \mathrm{P}<0.01,{ }^{* * *}, \mathrm{P}<0.001$ vs. MC903 + vehicle treatment group.

\section{Poly (I:C) treatment increases IgE production and dermal mast cell infiltration}

Serum IgE levels in patients with extrinsic $\mathrm{AD}$ are markedly elevated (33), and Th2-related cytokines including IL-4 and IL-13 can trigger B cells to produce $\operatorname{IgE}(34,35)$. An imbalance between Th1 and Th2 cytokines in patients with $\mathrm{AD}$ is thought to contribute to altered cell-mediated immune responses and IgE-mediated hypersensitivity, both of which are associated with the etiology of this disease (36). In light of these findings, we assessed $\operatorname{IgE}$ synthesis and mast cell infiltration in our model system, revealing MC903 treatment to increase $\operatorname{IgE}$ production (Figure $4 A$ ) and mast cell infiltration of the dermis (Figure $4 B$ ), with poly (I:C) treatment further enhancing these allergic inflammatory responses. 


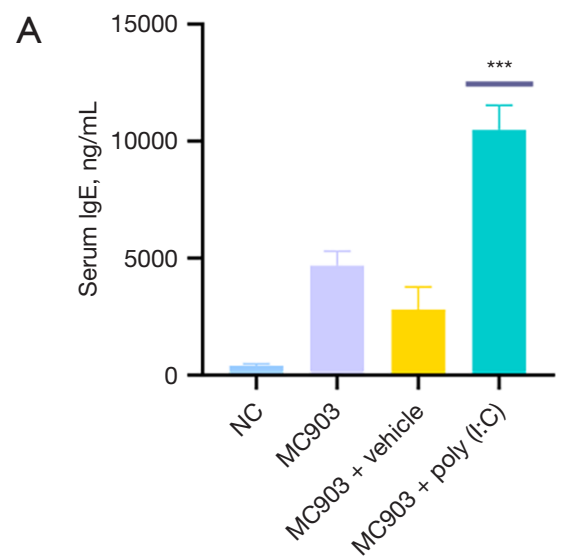

B

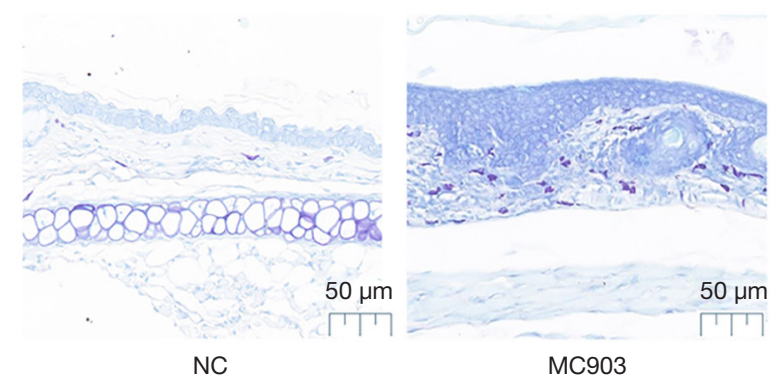

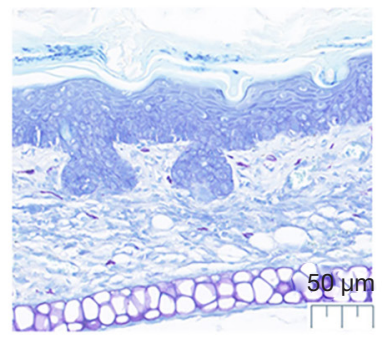

MC903 + vehicle

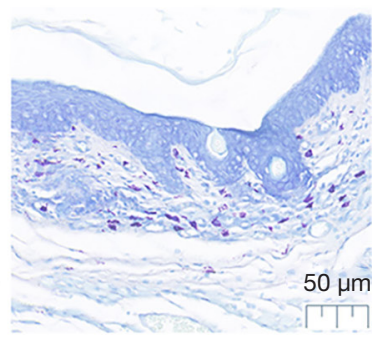

MC903 + poly $(\mathrm{l}: \mathrm{C})$

Figure 4 The impact of polyinosinic:polycytidylic acid [poly (I:C)] treatment on serum IgE was assessed in a murine model of MC903induced atopic dermatitis (AD)-like disease (A). Ear sections were stained with toluidine blue to demonstrate mast cell infiltration into the $\mathrm{AD}$-like cutaneous lesions. Scale bar $=50 \mu \mathrm{m}(\mathrm{B})$. Data are expressed as means $\pm \mathrm{SD}, \mathrm{n}=10 /$ group. ${ }^{* * *}, \mathrm{P}<0.001$ vs. MC903 + vehicle treatment group.

\section{Poly (I:C) administration increases the production of TSLP and IL-13 in a time-dependent fashion}

ELISAs revealed that serum IL-13 and TSLP levels rose in a time-dependent manner (Figure 5A,5B). Western blotting further revealed that continuous poly (I:C) injection for 4 days significantly increased TSLP and IL-13R $\alpha 1$ levels above those observed following a 0-1-day injection period, although peak levels of these cytokines appeared to be evident from approximately day 2 of treatment (Figure 5C-5F). Overall, these findings suggested that treatment with poly (I:C) can increase IL-13 and TSLP production in a timedependent fashion.

\section{Conclusions}

Prior work has highlighted a link between TLR2 and AD pathogenesis (26,37-39), but the role of TLR3 in this pathological context is not well understood. In previous reports, TLR3 ligands or cellular damage have been shown to drive keratinocytes to produce TSLP (27). In addition, poly (I:C) can stimulate primary human keratinocytes to produce TSLP (28), with the same also being true in $\mathrm{HaCaT}$ cells (40). While several cytokines (IL-4, IL-13, TNF- $\alpha$ ) and TLRs (TLR2, TLR5) can induce TSLP production, it remains unclear whether aberrant TLR3 activation is linked to AD pathogenesis (28). Therefore, more work is necessary to clarify how TLR3, TSLP, and other cytokines contribute to AD progression in vivo. While prior work has shown that TLR3 is associated with stimulating barrier repair $(41,42)$, our results suggest that exogenous activation of TLR 3 and consequent enhancement of TSLP expression can aggravate AD-related immunopathology.

This study explored how the systemic administration of poly (I:C) increased systemic TSLP expression (Figure 3 and Figure 5), as this cytokine serves as an important alarmin in AD. Our results revealed that poly (I:C) administration and TLR3 activation were associated with significantly increased TSLP levels in our murine model of MC903-induced 
A



C

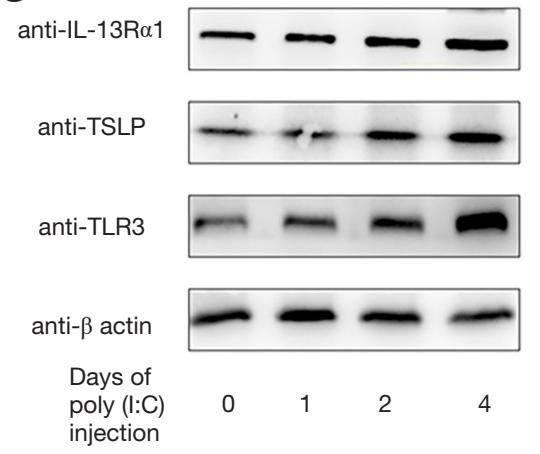

$E$



B

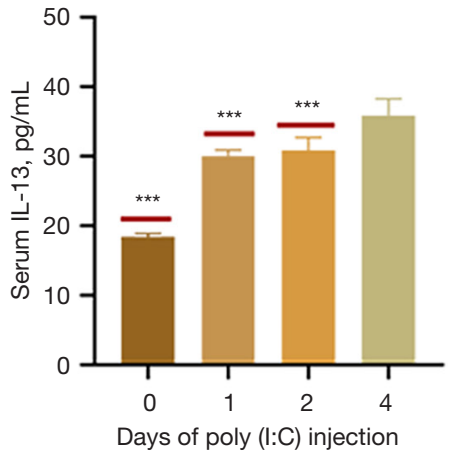

D

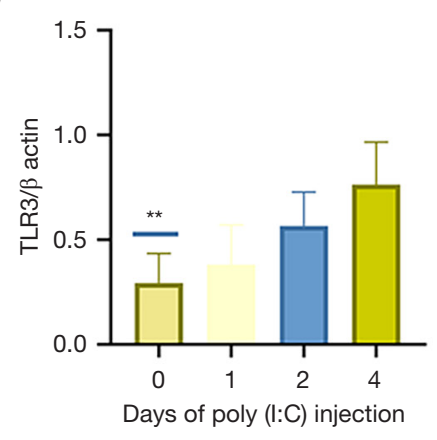

$\mathrm{F}$

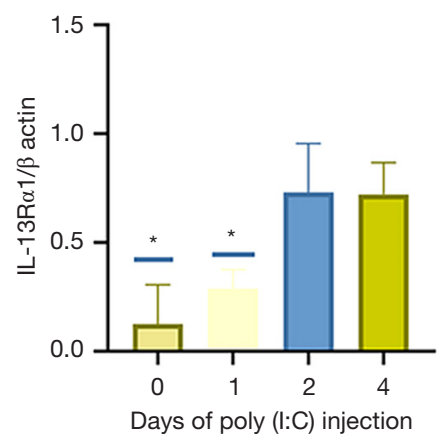

Figure 5 The impact of polyinosinic:polycytidylic acid [poly (I:C)] treatment on serum TSLP and IL-13 was assessed in a murine model of MC903-induced atopic dermatitis (AD)-like disease (A,B). Western blotting analyses of TLR3, TSLP, and IL-13Ra1 levels (C-F). Densitometric analyses are representative of 3 independent experiments shown in Figure $S 2$. Data are expressed as means \pm SD, in $(A, B)$, $\mathrm{n}=10 /$ group, in (C-F), $\mathrm{n}=3$ /group. ${ }^{*}, \mathrm{P}<0.05,{ }^{* *}, \mathrm{P}<0.01,{ }^{* * *}, \mathrm{P}<0.001$ vs. 4 days of poly (I:C) injection group.

AD-like disease. We additionally assessed serum IL-4 and IL-13 and tissue IL-13 levels, revealing similar trends without any apparent change in IFN- $\gamma$ expression (Figure 3). Given that IL-13 and associated signaling pathways are dominant in $\mathrm{AD}$-associated skin samples, whereas IL-4 expression is negligible, we focused specifically on IL-13 tissue levels (43). IL-13 signaling is regulated by the lowaffinity IL-13R $\alpha 1$ receptor, which can heterodimerize with
IL-4R $\alpha$ to generate a high-affinity IL-13 receptor that can signal through the JAK2/TYK2 proteins within cells (13). In contrast, the high-affinity IL-13R $\alpha 2$ receptor can readily bind to IL-13 but lacks cytoplasmic domain and thus does not act as a signaling mediator. Therefore, IL-13R $\alpha 1$ exerts pathogenic activity in $\mathrm{AD}$ whereas IL-13R $\alpha 2$ can serve as a decoy receptor $(13,44)$. There is also recent evidence of IL-13R $\alpha 2$ overexpression in $\mathrm{AD}$ skin lesions, and it may 
function as a negative regulator of allergic inflammatory responses induced by IL-13 in a protective capacity (4547). In our Western blotting and immunohistochemical experiments, we evaluated changes in IL-13R $\alpha 1$ reflecting IL-13-related tissue activity. Together, these results suggested the ability of TLR 3 to activate Th2 cytokine signaling, which is central to the pathogenesis of $\mathrm{AD}$.

Both IL-4 and IL-13 play central roles in inducing and perpetuating type 2 inflammatory responses that culminate in $\operatorname{IgE}$ production $(48,49)$. As $\operatorname{IgE}$ and antigens can activate mast cells, we next explored the ability of poly (I:C) to enhance IgE production and dermal mast cell infiltration in our mouse model system. Poly (I:C) significantly enhanced $\mathrm{IgE}$ expression (Figure 4A), and TBO staining further demonstrated that it could enhance IgE-derived mast cell infiltration (Figure 4B). These findings coincided well with observed changes in skin lesions (Figure $4 B$ ), bodyweight (Figure 2C) and ear thickness (Figure 2D), and the results of $\mathrm{H} \& \mathrm{E}$ staining assays (Figure $2 B$ ).

To additionally demonstrate the impact of poly (I:C) treatment and TLR3 activation on the expression of IL-13 and TSLP, we treated MC903-induced AD-like model mice with poly (I:C) for 0-4 days in different treatment groups (Figure 1B). Overall the results suggested that poly (I:C) could enhance IL-13 and TSLP production in a time-dependent manner, although the Western blotting results were not robust (Figure 5).

This study found that exogenously activating TLR3 can promote increases in serum and tissue TSLP expression. Thus, TLR3 may become a novel potential biomarker and therapeutic target for $\mathrm{AD}$. Using an in vivo animal model system, we confirmed that systemic poly (I:C) administration was enough to induce IL-4 and IL-13 expression, exacerbating skin lesions in treated mice. While these findings offer clear clinical relevance, we did not demonstrate that TLR3 activation may play a natural role in $\mathrm{AD}$, and did not explore related signaling pathways. We posit that targeting the TLR3 pathway may represent a promising therapeutic approach for patients with $\mathrm{AD}$, although additional work will be necessary to confirm the functional and clinical importance of TLR3 in the context of $\mathrm{AD}$ development.

\section{Acknowledgments}

Funding: This work was partially supported by Nanjing Medical Science and Technology Development Project (Grant No. YKK16134).

\section{Footnote}

Reporting Checklist: The authors have completed the ARRIVE reporting checklist. Available at https://atm. amegroups.com/article/view/10.21037/atm-22-282/rc

Data Sharing Statement: Available at https://atm.amegroups. com/article/view/10.21037/atm-22-282/dss

Conflicts of Interest: All authors have completed the ICMJE uniform disclosure form (available at https:// atm.amegroups.com/article/view/10.21037/atm-22-282/ coif). All authors report funding from Nanjing Medical Science and Technology Development Project (Grant No. YKK16134). The authors have no other conflicts of interest to declare.

Ethical Statement: The authors are accountable for all aspects of the work in ensuring that questions related to the accuracy or integrity of any part of the work are appropriately investigated and resolved. All animal studies were approved by the Institutional Ethics Committee of Nanjing First Hospital, Nanjing Medical University (Project License DWSY-2001134), and all experimentally related processes and animal care followed the institutional ethics directions for animal-related experimental processes.

Open Access Statement: This is an Open Access article distributed in accordance with the Creative Commons Attribution-NonCommercial-NoDerivs 4.0 International License (CC BY-NC-ND 4.0), which permits the noncommercial replication and distribution of the article with the strict proviso that no changes or edits are made and the original work is properly cited (including links to both the formal publication through the relevant DOI and the license). See: https://creativecommons.org/licenses/by-nc-nd/4.0/.

\section{References}

1. Nakahara T, Kido-Nakahara M, Tsuji G, et al. Basics and recent advances in the pathophysiology of atopic dermatitis. J Dermatol 2021;48:130-9.

2. Navinés-Ferrer A, Serrano-Candelas E, Molina-Molina GJ, et al. IgE-Related Chronic Diseases and Anti-IgEBased Treatments. J Immunol Res 2016;2016:8163803.

3. Weidinger S, Beck LA, Bieber T, et al. Atopic dermatitis. Nat Rev Dis Primers 2018;4:1. 
4. Munera-Campos M, Carrascosa JM. Innovation in Atopic Dermatitis: From Pathogenesis to Treatment. Actas Dermosifiliogr (Engl Ed) 2020;111:205-21.

5. Ferreira S, Guttman-Yassky E, Torres T. Selective JAK1 Inhibitors for the Treatment of Atopic Dermatitis: Focus on Upadacitinib and Abrocitinib. Am J Clin Dermatol 2020;21:783-98.

6. Rodrigues MA, Torres T. JAK/STAT inhibitors for the treatment of atopic dermatitis. J Dermatolog Treat 2020;31:33-40.

7. Sugaya M. The Role of Th17-Related Cytokines in Atopic Dermatitis. Int J Mol Sci 2020;21:1314.

8. Matsunaga MC, Yamauchi PS. IL-4 and IL-13 Inhibition in Atopic Dermatitis. J Drugs Dermatol 2016;15:925-9.

9. Chiricozzi A, Maurelli M, Peris K, et al. Targeting IL-4 for the Treatment of Atopic Dermatitis. Immunotargets Ther 2020;9:151-6.

10. Bieber T. Interleukin-13: Targeting an underestimated cytokine in atopic dermatitis. Allergy 2020;75:54-62 .

11. Bao K, Reinhardt RL. The differential expression of IL-4 and IL-13 and its impact on type-2 immunity. Cytokine 2015;75:25-37.

12. Harb H, Chatila TA. Mechanisms of Dupilumab. Clin Exp Allergy 2020;50:5-14.

13. Furue K, Ito T, Tsuji G, et al. The IL-13-OVOL1-FLG axis in atopic dermatitis. Immunology 2019;158:281-6.

14. Wollenberg A, Weidinger S, Worm M, et al. Tralokinumab in atopic dermatitis. J Dtsch Dermatol Ges 2021;19:1435-42.

15. Li M, Hener P, Zhang Z, et al. Topical vitamin D3 and low-calcemic analogs induce thymic stromal lymphopoietin in mouse keratinocytes and trigger an atopic dermatitis. Proc Natl Acad Sci U S A 2006;103:11736-41.

16. Li M, Hener P, Zhang Z, et al. Induction of thymic stromal lymphopoietin expression in keratinocytes is necessary for generating an atopic dermatitis upon application of the active vitamin D3 analogue MC903 on mouse skin. J Invest Dermatol 2009;129:498-502.

17. Moosbrugger-Martinz V, Schmuth M, Dubrac S. A Mouse Model for Atopic Dermatitis Using Topical Application of Vitamin D3 or of Its Analog MC903. Methods Mol Biol 2017;1559:91-106.

18. Varricchi G, Pecoraro A, Marone G, et al. Thymic Stromal Lymphopoietin Isoforms, Inflammatory Disorders, and Cancer. Front Immunol 2018;9:1595.

19. Corren J, Ziegler SF. TSLP: from allergy to cancer. Nat Immunol 2019;20:1603-9.

20. Leyva-Castillo JM, Hener P, Michea P, et al. Skin thymic stromal lymphopoietin initiates Th2 responses through an orchestrated immune cascade. Nat Commun 2013;4:2847.

21. Roan F, Obata-Ninomiya K, Ziegler SF. Epithelial cellderived cytokines: more than just signaling the alarm. J Clin Invest 2019;129:1441-51.

22. Wilson SR, Thé L, Batia LM, et al. The epithelial cellderived atopic dermatitis cytokine TSLP activates neurons to induce itch. Cell 2013;155:285-95.

23. Fitoussi J, Virassamynaïk S, Callejon S, et al. Inhibition of thymic stromal lymphopoietin production to improve pruritus and quality of life in infants and children with atopic dermatitis. J Cosmet Dermatol 2020;19:2061-9.

24. Schwartz C, Eberle JU, Hoyler T, et al. Opposing functions of thymic stromal lymphopoietin-responsive basophils and dendritic cells in a mouse model of atopic dermatitis. J Allergy Clin Immunol 2016;138:14431446.e8.

25. Simpson EL, Parnes JR, She D, et al. Tezepelumab, an anti-thymic stromal lymphopoietin monoclonal antibody, in the treatment of moderate to severe atopic dermatitis: A randomized phase 2a clinical trial. J Am Acad Dermatol 2019;80:1013-21.

26. Sun L, Liu W, Zhang LJ. The Role of Toll-Like Receptors in Skin Host Defense, Psoriasis, and Atopic Dermatitis. J Immunol Res 2019;2019:1824624.

27. Kinoshita H, Takai T, Le TA, et al. Cytokine milieu modulates release of thymic stromal lymphopoietin from human keratinocytes stimulated with double-stranded RNA. J Allergy Clin Immunol 2009;123:179-86.

28. Xie Y, Takai T, Chen X, et al. Long TSLP transcript expression and release of TSLP induced by TLR ligands and cytokines in human keratinocytes. J Dermatol Sci 2012;66:233-7.

29. Takai T, Chen X, Xie Y, et al. TSLP expression induced via Toll-like receptor pathways in human keratinocytes. Methods Enzymol 2014;535:371-87.

30. Kubo T, Kamekura R, Kumagai A, et al. $\Delta$ Np63 controls a TLR3-mediated mechanism that abundantly provides thymic stromal lymphopoietin in atopic dermatitis. PLoS One 2014;9:e105498.

31. Liu XJ, Mu ZL, Zhao Y, et al. Topical Tetracycline Improves MC903-induced Atopic Dermatitis in Mice through Inhibition of Inflammatory Cytokines and Thymic Stromal Lymphopoietin Expression. Chin Med J (Engl) 2016;129:1483-90.

32. Elias PM, Wakefield JS. Therapeutic implications of a barrier-based pathogenesis of atopic dermatitis. Clin Rev Allergy Immunol 2011;41:282-95. 
33. Bruijnzeel-Koomen C, van Reysen F, Mudde GC. IgE and atopic dermatitis. Clin Exp Allergy 1991;21 Suppl 1:294-301.

34. Van der Pouw Kraan TC, Van der Zee JS, Boeije LC, et al. The role of IL-13 in IgE synthesis by allergic asthma patients. Clin Exp Immunol 1998;111:129-35.

35. Deo SS, Mistry KJ, Kakade AM, et al. Role played by Th2 type cytokines in IgE mediated allergy and asthma. Lung India 2010;27:66-71.

36. David Boothe W, Tarbox JA, Tarbox MB. Atopic Dermatitis: Pathophysiology. Adv Exp Med Biol 2017;1027:21-37.

37. Panzer R, Blobel C, Fölster-Holst R, et al. TLR2 and TLR4 expression in atopic dermatitis, contact dermatitis and psoriasis. Exp Dermatol 2014;23:364-6.

38. Tyurin YA, Shamsutdinov AF, Kalinin NN, et al. Association of Toll-Like Cell Receptors TLR2 (p.Arg753GLN) and TLR4 (p.Asp299GLY) Polymorphisms with Indicators of General and Local Immunity in Patients with Atopic Dermatitis. J Immunol Res 2017;2017:8493545.

39. Kaesler S, Volz T, Skabytska Y, et al. Toll-like receptor 2 ligands promote chronic atopic dermatitis through IL-4mediated suppression of IL-10. J Allergy Clin Immunol 2014;134:92-9.

40. Lee KH, Cho KA, Kim JY, et al. Filaggrin knockdown and Toll-like receptor 3 (TLR3) stimulation enhanced the production of thymic stromal lymphopoietin (TSLP) from epidermal layers. Exp Dermatol 2011;20:149-51.

41. Borkowski AW, Kuo IH, Bernard JJ, et al. Tolllike receptor 3 activation is required for normal skin

Cite this article as: Wan H, Yang H, Wei M, Chen W. Polyinosinic:polycytidylic acid aggravates calcipotriol-induced atopic dermatitis-like skin lesions in mice by increasing the expression of thymic stromal lymphopoietin. Ann Transl Med 2022;10(4):209. doi: 10.21037/atm-22-282 barrier repair following UV damage. J Invest Dermatol 2015;135:569-78.

42. Feingold KR. TLR3: a receptor that recognizes cell injury is essential for permeability barrier homeostasis following UV irradiation. J Invest Dermatol 2015;135:339-40.

43. Tsoi LC, Rodriguez E, Degenhardt F, et al. Atopic Dermatitis Is an IL-13-Dominant Disease with Greater Molecular Heterogeneity Compared to Psoriasis. J Invest Dermatol 2019;139:1480-9.

44. Ranasinghe C, Trivedi S, Wijesundara DK, et al. IL-4 and IL-13 receptors: Roles in immunity and powerful vaccine adjuvants. Cytokine Growth Factor Rev 2014;25:437-42.

45. Ulzii D, Kido-Nakahara M, Nakahara T, et al. Scratching Counteracts IL-13 Signaling by Upregulating the Decoy Receptor IL-13R $\alpha 2$ in Keratinocytes. Int J Mol Sci 2019;20:3324.

46. Guttman-Yassky E, Bissonnette R, Ungar B, et al. Dupilumab progressively improves systemic and cutaneous abnormalities in patients with atopic dermatitis. J Allergy Clin Immunol 2019;143:155-72.

47. Furue M, Ulzii D, Nakahara T, et al. Implications of IL-13R $\alpha 2$ in atopic skin inflammation. Allergol Int 2020;69:412-6.

48. Gandhi NA, Bennett BL, Graham NM, et al. Targeting key proximal drivers of type 2 inflammation in disease. Nat Rev Drug Discov 2016;15:35-50.

49. Gandhi NA, Pirozzi G, Graham NMH. Commonality of the IL-4/IL-13 pathway in atopic diseases. Expert Rev Clin Immunol 2017;13:425-37.

(English Language Editor: LJ. Roberts) 




Figure S1 Western blotting analyses of TLR3, TSLP, and IL-13R 1 levels. Calcipotriol (MC903) + polyinosinic:polycytidylic acid [poly (I:C)] treatment increased TLR3, TSLP, and IL-13R 1 levels relative to MC903 + vehicle treatment.

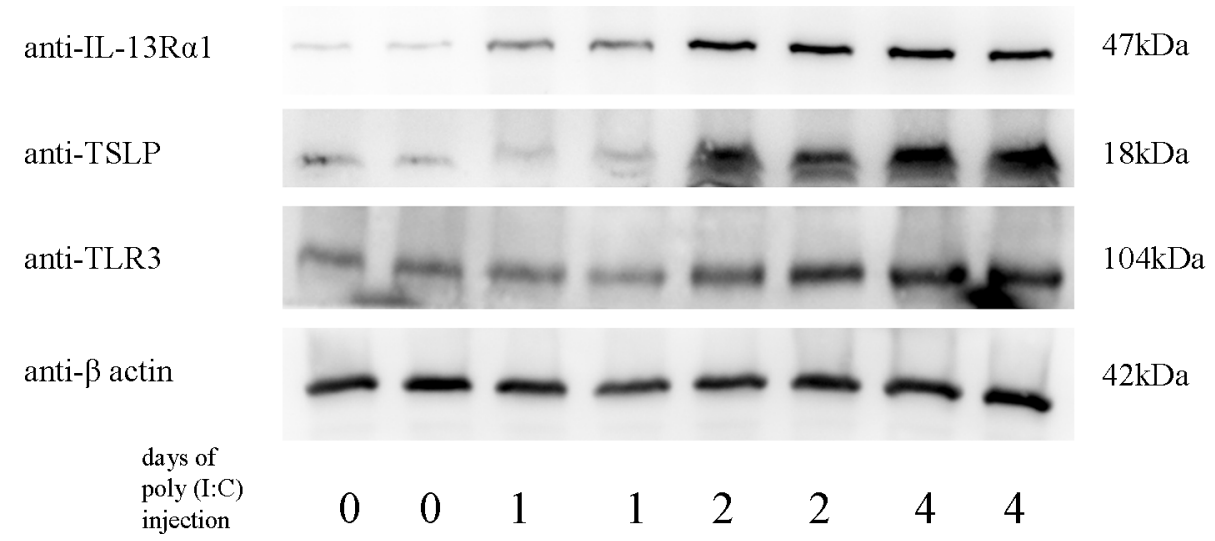

Figure S2 Western blotting analyses of TLR3, TSLP, and IL-13R $\alpha 1$ levels. Polyinosinic:polycytidylic acid [poly (I:C)] administration four consecutive days significantly increased levels of TSLP and IL-13R $\alpha 1$ as compared to injection for $0-1$ days. 